\title{
On a Cercopithifilaria sp. transmitted by Rhipicephalus sanguineus: a neglected, but widespread filarioid of dogs
}

Domenico Otranto ${ }^{1 *}$, Emanuele Brianti ${ }^{2}$, Maria Stefania Latrofa ${ }^{1}$, Giada Annoscia' ${ }^{1}$ Stefania Weigl ${ }^{1}$, Riccardo Paolo Lia', Gabriella Gaglio², Ettore Napoli², Salvatore Giannetto², Elias Papadopoulos ${ }^{3}$, Guadalupe Mirò ${ }^{4}$, Filipe Dantas-Torres ${ }^{1}$ and Odile Bain ${ }^{5}$

\begin{abstract}
Background: This study was aimed at investigating the distribution of a Cercopithifilaria sp. sensu Otranto et al., 2011 with dermal microfilariae recently identified in a dog from Sicily (Italy). A large epidemiological survey was conducted by examining skin samples $(n=917)$ and ticks $(n=890)$ collected from dogs at different time points in Italy, central Spain and eastern Greece.

Results: The overall prevalence of Cercopithifilaria sp. in the sampled animal populations was $13.9 \%$ and $10.5 \%$ by microscopy of skin sediments and by PCR on skin samples, respectively. Up to $21.6 \%$ and $45.5 \%$ of dogs in Spain were positive by microscopical examination and by PCR. Cumulative incidence rates ranging from $7.7 \%$ to $13.9 \%$ were estimated in dogs from two sites in Italy. A low level of agreement between the two diagnostic tests (microscopical examination and PCR) was recorded in sites where samples were processed in parallel. Infestation rate as determined by tick dissection (from $5.2 \%$ to $16.7 \%$ ) was higher than that detected by PCR (from $0 \%$ to 3.9\%); tick infestation was significantly associated with Cercopithifilaria sp. infestation in dogs from two out of four sites. Developing larvae found in ticks were morphometrically studied and as many as 1469 larvae were found in a single tick.

Conclusions: Our data suggest that, in addition to the most common species of filarioids known to infest dogs (i. e., Dirofilaria immitis, Dirofilaria repens and Acanthocheilonema reconditum), Cercopithifilaria sp. with dermal microfilariae should be considered due to its widespread distribution in southern Europe and high frequency in tick-exposed dogs.
\end{abstract}

Keywords: canine filarioids, Cercopithifilaria, Cercopithifilaria bainae, Cercopithifilaria grassii, Rhipicephalus sanguineus, vector, epidemiology, Europe

\section{Background}

Among veterinarians and parasitologists, canine filariae with haematic microfilariae (e.g., Dirofilaria immitis, Dirofilaria repens, Acanthocheilonema reconditum and Acanthocheilonema dracunculoides) are known better than those with only dermal microfilariae (e.g., Onchocerca lupi and Cercopithifilaria spp.). This is probably due to the fact that dermal filariae cause limited or no

\footnotetext{
* Correspondence: d.otranto@veterinaria.uniba.it

'Dipartimento di Sanità Pubblica e Zootecnia, Università degli Studi di Bari, Valenzano, BA, Italy

Full list of author information is available at the end of the article
}

clinical alterations (with the exception of O. lupi) and that skin samples are difficult to collect since this procedure is invasive and thus not easily accepted by pet owners. Additionally, blood microfilariae are easily visible in blood smears during routine examination of dogs. However, cases of O. lupi causing acute or chronic ocular disease in dogs (i.e., conjunctivitis, photophobia, lacrimation, ocular discharge, exophthalmia) [1] have been reported in the United States [2-4], and Europe [5-8]. Likewise for many other filarioids [9], O. lupi has been recently been implicated as an agent of ocular zoonosis [10].

\section{Biomed Central}


Filarioids belonging to the genus Cercopithifilaria Eberhard, 1980, which are transmitted by hard ticks (Ixodidae), parasitize a range of host species, including dogs [11]. Recently, dermal microfilariae of the genus Cercopithifilaria found in a dog from Sicily (southern Italy), Cercopithifilaria sp. sensu Otranto et al., 2011 (hereinafter reported as Cercopithifilaria sp.), were characterised morphologically and differentiated from other microfilariae commonly found in dogs [12]. The genetic identity of these microfilariae was also assessed by molecular amplification, sequencing and by a comparative phylogenetic analysis of multiple ribosomal ITS-2 and mitochondrial (cox1 and 12S) target genes [12]. Interestingly, the microfilariae examined in Sicily were short (mean length, 185 $\mu \mathrm{m})$, and thus distinct from Cercopithifilaria grassii, a parasite described in a dog from central Italy, more than one century ago as Filaria grassii [13], which had exceptionally long microfilariae $(660 \mu \mathrm{m})$. The microfilariae of Cercopithifilaria sp. had a similar size to those of a species described in Brazilian dogs, Cercopithifilaria bainae Almeida \& Viente, 1984 [14]. However, since neither adult nematodes from the Sicilian case were available nor the holotype of the Brazilian species was restudied for comparison, a conclusive specific assessment of Cercopithifilaria sp. [12] is lacking. Nevertheless, the competence of the brown dog tick, Rhipicephalus sanguineus, as an intermediate host of this filarial species has been experimentally investigated and results obtained showed that these microfilariae develop to the third-stage infective larvae (L3) in nymphs [15]. Additionally, a PCR protocol for the detection of Cercopithifilaria sp. in dog skin samples and ticks was assessed and proposed as a tool for further epidemiological studies [12].

In past years, single reports of filarioids identified as $C$. grassii were described in ticks from Switzerland [16] and northern Italy [17] but no data on the distribution of Cercopithifilaria spp. in dogs and in its tick vectors are so far available in the literature. This lack of knowledge impairs the understanding of the epidemiology of Cercopithifilaria spp. infecting dog populations and the study of their pathogenic role to dogs, at a local (dermic) or systemic level. Thus, one year after the retrieval of the first case of Cercopithifilaria sp. infestation in a dog from Sicily [12], this current study was carried out to investigate the occurrence of this filarioid in selected populations of dogs exposed to $R$. sanguineus from three countries of the Mediterranean area (i.e., Italy, Spain and Greece). Skin samples from dogs enrolled in previous studies at different time points or specifically sampled from animals together with their ticks were tested microscopically and/or molecularly in order to give the first comprehensive account of the occurrence of this Cercopithifilaria sp. in dogs living in countries in the Mediterranean area.

\section{Materials and methods}

\section{Study areas and sampling times}

A total of 917 skin samples were collected at different time points from dogs living in regions of southern Italy $(\mathrm{n}=843)$, central Spain $\mathrm{n}=51$ (site F) and eastern Greece $n=23$ (site G). Animals from Italy come from Apulia (municipalities of Bari $\mathrm{n}=280$ (site A), Ginosa $\mathrm{n}$ $=320$ (site B) and Putignano $\mathrm{n}=80$ (site C)), Basilicata (Parco Regionale di Gallipoli Cognato - Piccole Dolomiti Lucane $\mathrm{n}=50$ (site D)) and Sicily (municipalities of Palermo and Messina $\mathrm{n}=113$ (site E)) (Figure 1).

Skin samples from Apulia (680) were collected under the context of previous studies aiming to perform a molecular diagnosis of Leishmania infantum [18,19]. In particular, 600 skin samples were sampled from dogs on March 2005 from site A (280) and B (320), while the remaining 80 animals were sampled on October 2009 from site $C$. In addition, in order to evaluate the incidence rate of Cercopithifilaria sp. infestation in the study populations, skin samples of some dogs were sampled again at 7 and 10 months intervals in sites B and $C$, respectively.

Dogs from other sites were sampled between February 2011 (site E) and July 2011 (site D), specifically for this study. All dogs sampled were naturally exposed to $R$. sanguineus infestation throughout the year [20-22] and mainly during spring and summer, which are the seasons with the higher number of ticks infesting dogs in temperate regions [23]. All animals sampled were not treated with endo- or ecto-parasiticide. During the collection of skin samples, dogs were also checked for tick presence and a total $890 R$. sanguineus ticks, almost all adults but also some nymphs, were individually collected from animals sampled on site $C(\mathrm{n}=257)$, site $\mathrm{D}(\mathrm{n}=$ $77)$, site $E(n=272)$, site $F(n=66)$ and site $G(n=$ 218).

The study design and the experimental procedures for skin samples collected from previous studies (i.e., sites A, B and C) were approved by the Animal Ethics Committee of the University of Bari (Italy) and authorized by the Italian Ministry of Health (authorization number 72/ 2009C, $n^{\circ}$. 69062; 11/28/2008) [18,19].

\section{Sampling and laboratory procedures}

Due to the different provenance and timing of sampling, the procedures varied slightly as detailed in the following. Skin samples were collected using a disposable scalpel from the right shoulder or inter-scapular region (about $0.5 \times 0.5 \times 0.6 \mathrm{~cm}$ ) and stored directly at $-20^{\circ} \mathrm{C}$ (sites $\mathrm{A}, \mathrm{B}$, and $\mathrm{C}$ ) or soaked in saline solution for 10 min at $37^{\circ} \mathrm{C}$ (sites $\mathrm{D}, \mathrm{E}, \mathrm{F}$, and $\mathrm{G}$ ) and thereafter removed and stored at $-20^{\circ} \mathrm{C}$ for the molecular analysis. When skin samples were soaked in saline solution, the 


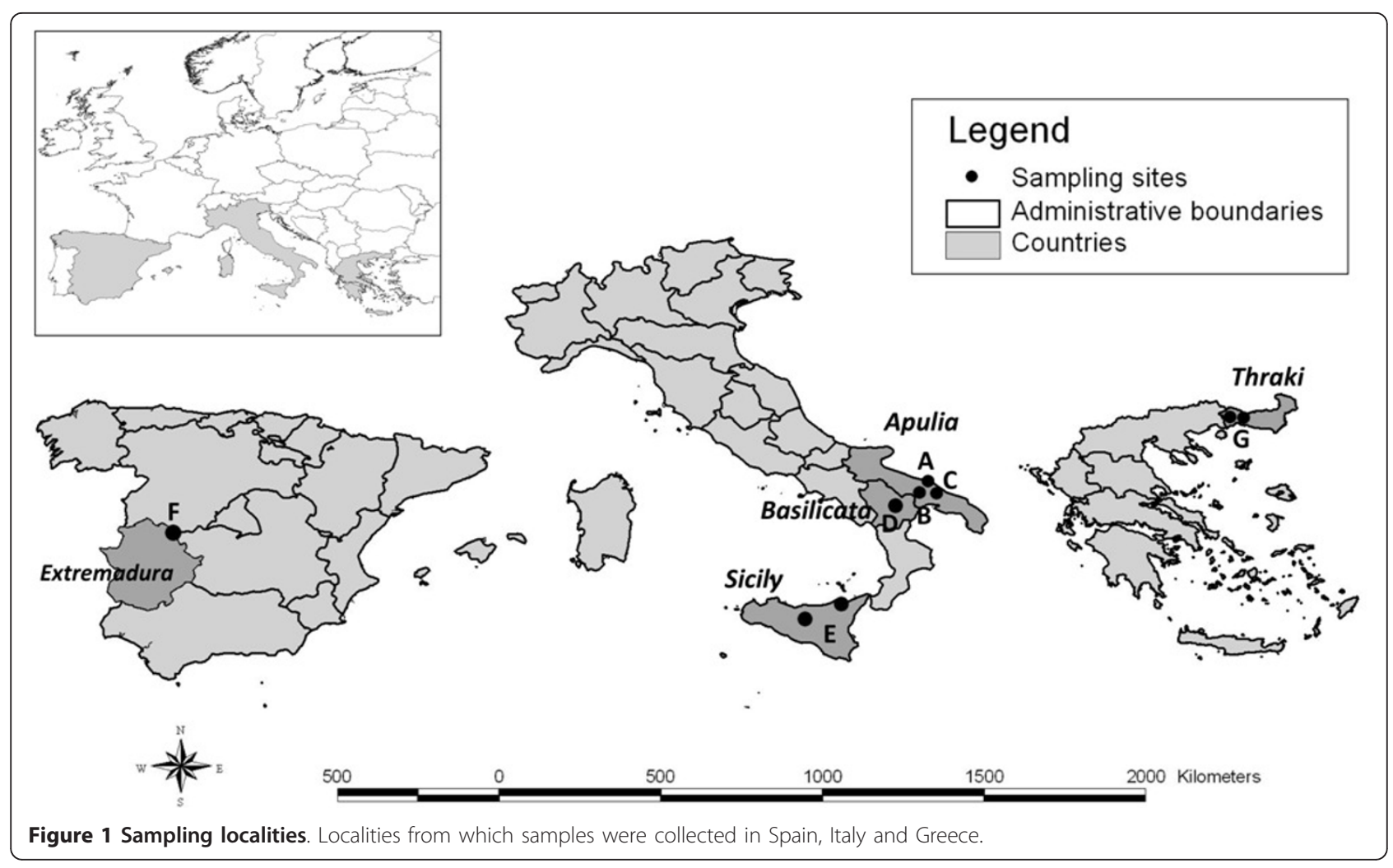

sediment was observed under light microscopy (two fields of $10 \times 10 \mathrm{~mm}$ coverslip each) after adding a drop of methylene blue (1\%) whereas samples from Sites A, $\mathrm{B}$, and $\mathrm{C}$ were tested by PCR only. Dermal microfilariae were morphologically identified [12]. Briefly, microfilariae have a rounded head and a short dorso-ventrally flattened body (mean length of $186.7 \pm 3.9 \mu \mathrm{m}$, width 8.5-11 $\mu \mathrm{m}$ and 3-3.5 wide in lateral view). No sheath is present and the body cuticle is thick with conspicuous transverse striations. Approximately 4-5 angular nuclei are detectable in dorso-ventral view and one rounded nucleus in lateral view on a transverse line.

Ticks collected were placed on a microscope slide and individually processed in a few drops of sterile saline solution under a stereomicroscope. Once ticks have been dissected, a drop of methylene blue (1\%) was added to visualize the larval nematode and a coverslip was placed on the preparation. This preparation was microscopically observed at $10 \times$ and $40 \times$ magnification. Larvae recovered in ticks were morphologically identified [15]; furthermore, developing forms found in ticks collected in sites D and E were also counted and staged. Following the microscopical observation and irrespective of the positivity, the remaining part of dissected ticks was stored in individual vials containing $70 \%$ ethanol for further molecular analysis (see below). Ticks collected from dogs in site $\mathrm{C}$ were molecularly processed only. Microfilariae found in dermal samples and developing forms found in the dissected ticks were photographed with a digital camera (Zeiss Axiocam MRc, Carl Zeiss AG, Germany) mounted on the microscope (Zeiss Axioscop 2 plus, Carl Zeiss AG, Germany) and measurements (in micrometres) were taken with the AxioVision rel. 4.8 software (Carl Zeiss AG, Germany).

\section{Molecular examination}

Genomic DNA was extracted from skin samples using a commercial kit (DNeasy Blood \& Tissue Kit, Qiagen, $\mathrm{GmbH}$, Hilden, Germany) in accordance with the manufacturer's instructions, whereas from $R$. sanguineus adult ticks as described elsewhere [24]. Skin samples and ticks were individually tested. All samples were molecularly processed for specific amplification of a partial cox 1 ( 304 bp) gene fragment targeting Cercopithifilaria sp., using specific primers CbCox1F/NTR following reaction procedures and amplification protocol described in the literature [12]. DNA from $R$. sanguineus, blood and skin samples from laboratory-reared beagles [25] were used as negative controls, along with a no DNA sample, which were included in each reaction to test the specificity of the reaction and to assess the presence of contaminants. About $20 \%$ of amplicons were purified using Ultrafree-DA columns (Amicon, Millipore; Bedford, 
USA) and sequenced directly using the Taq DyeDeoxyTerminator Cycle Sequencing Kit (v.2, Applied Biosystems) in an automated sequencer (ABI-PRISM 377). Sequences were aligned using the ClustalW program [26] and compared among them and with those available in GenBank ${ }^{\mathrm{TM}}$ dataset by BLAST analysis.

\section{Data analysis}

Differences in the frequencies of Cercopithifilaria infestation both in dogs or ticks in the same study site were evaluated for statistical significance by using chi-square test (with Yates' correction) or, when appropriate, by Fisher's exact test. Cumulative incidence for Cercopithifilaria sp. in animals from sites B and C was calculated as the proportion of new cases (molecularly detected) that occur in the two study populations over the period of months at risk [27]. Agreement between the two diagnostic tests was evaluated using Kappa (K) statistics and the proportion of agreement beyond chance expressed as values of $\mathrm{K}$ in a scale ranging from 0 (no agreement) to 1 (perfect agreement). The association between Cercopithifilaria infestation in dogs and in ticks was expressed by the odds ratio (OR) and the hypothesis tested for statistical significance by Fisher's exact test. Critical significance level $(\alpha)$ was set at 5\% (0.05) and all tests were performed two-sided. Statistical analyses was carried out using the statistical packages GraphPad InStat v. 3.05 (GraphPad Software, Inc.) and WinEpiscope 2.0.

\section{Results}

A total of 237 skin sediments and 877 skin samples were examined for the presence of Cercopithifilaria sp. by microscopic and molecular analysis, respectively.
Based on the diagnostic test employed, the overall prevalence of Cercopithifilaria sp. in the sampled animal populations ranged from $13.9 \%$ to $10.5 \%$ by using microscopy and PCR, respectively. The higher prevalence rate of infested animals was recorded in Spain either by microscopical examination of skin sediments $(21.6 \%)$ or by molecular detection on skin samples (45.5\%) whereas the lower positivity rate was in Greece (4.3\%). In Italy, according to the sites and to the diagnostic tests employed, the prevalence of Cercopithifilaria sp. infestation in dogs varied from $5.3 \%$ (site B) up to $19.5 \%$ in site $\mathrm{E}$ (Table 1).

No difference was detected in the frequency of positive samples from the same site as determined by the two diagnostic methods except in site $\mathrm{D}(P=0.0266)$ where PCR produced no positive result. A significant difference in the frequency of infection between dog sexes was only found in site A where male dogs showed a higher rate $(23.4 \%)$ of infestation than females $(7.5 \% ; P=0.0003)$. The incidence of infestation was calculated in dogs from sites B and C. Indeed, a cumulative incidence of 7.7\% (8 out 104 animals over 10 months at risk) and of $13.9 \%$ (5 out 36 animals over 7 months at risk) was estimated in sites $B$ and $C$, respectively (data not shown).

A low level of agreement between the two diagnostic tests was recorded in sites where samples were tested by microscopy and PCR. The higher proportion of agreement $(\mathrm{K}=0.241)$ was recorded in samples from site $(\mathrm{F})$ where $2 / 5$ of PCR-positive samples were also positive by microscopy and $1 / 6$ of PCR-negative samples were positive by microscopical examination. In site E, six out of 22 PCRpositive samples were also positive by microscopical examination, whereas 9 out of 91 PCR-negative samples were positive by microscopical examination $(K=0.198)$ (Table

Table 1 Number and percentage (in brackets) of dogs and ticks positive for Cercopithifilaria sp. divided according to the geographical site of collection and diagnostic method used (i.e., microscopical examination of skin sediment or molecular analysis of skin samples)

\begin{tabular}{|c|c|c|c|c|c|c|c|}
\hline \multirow{3}{*}{ Country } & \multirow{3}{*}{$\begin{array}{l}\text { Locality/ } \\
\text { Region }\end{array}$} & \multicolumn{3}{|l|}{ Dogs } & \multicolumn{3}{|l|}{ Ticks } \\
\hline & & Microscopical examination & Molecular analysis & $P$ & Microscopical examination & Molecular analysis & $P$ \\
\hline & & pos/tot (\%) & pos/tot (\%) & & pos/tot (\%) & pos/tot (\%) & \\
\hline \multicolumn{8}{|l|}{ Italy } \\
\hline & Bari (A) & - & $38 / 280(13.6 \%)$ & - & - & - & - \\
\hline & Ginosa (B) & - & $17 / 320(5.3 \%)$ & - & - & - & - \\
\hline & Putignano (C) & - & $10 / 80(12.5 \%)$ & - & - & 10/257 (3.9\%) & - \\
\hline & Basilicata (D) & $6 / 50(12 \%)$ & $0 / 50(0 \%)$ & $0.026^{*}$ & $4 / 77(5.2 \%)$ & $2 / 77(2.6 \%)$ & $0.6812^{*}$ \\
\hline & Sicilia (E) & 15/113 (13.3\%) & $22 / 113(19.5 \%)$ & 0.2808 & $41 / 272(15.1 \%)$ & $7 / 272(2.6 \%)$ & $<0.0001$ \\
\hline \multicolumn{8}{|l|}{ Spain } \\
\hline & La Vera $(F)$ & $11 / 51(21.6 \%)$ & $5 / 11(45.4 \%)$ & $0.132^{*}$ & 11/66 (16.7\%) & 0/66 (0\%) & 0.0016 \\
\hline \multicolumn{8}{|l|}{ Greece } \\
\hline & Xanthi (G) & $1 / 23(4.3 \%)$ & $0 / 23(0 \%)$ & $1^{*}$ & $14 / 218$ (6.4\%) & $1 / 218(0.5 \%)$ & 0.0016 \\
\hline
\end{tabular}

$P$ values indicate the statistical significance of differences between test frequencies as determined by Yates' corrected chi-square test or Fisher's exact test ${ }^{*}$ ) when appropriate $(\alpha=0.05)$. 
2). No concordance was observed between the two diagnostic tests in sites D and G, where all the samples processed by PCR were negative.

Prevalence of infestation in ticks collected ranged from 5.2 to $16.7 \%$ and from $0 \%$ to $3.9 \%$ by using dissection and PCR, respectively (Table 1 ). In all cases, the positivity rate determined by tick dissection was higher than that by molecular examination. With the exception of site D, prevalence of infested ticks determined by microscopy was correlated with those obtained by the microscopical examination of skin sediments of dogs from the same site. In addition, tick infestation was significantly associated with Cercopithifilaria sp. infestation in dogs from sites $\mathrm{D}(\mathrm{OR}=11.9, P=0.0184)$ and $\mathrm{E}(\mathrm{OR}=16.2, P<$ 0.0001 ) (Table 3). Infected ticks were collected from both positive (38/90, 42.2\%) and negative (52/90, 57.8\%) dogs. All developing forms found in ticks were consistent with those of Cercopithifilaria sp. at different stages described (Figure 2). Larval forms detected in ticks were counted and staged in 45 positive ticks (i.e., 4 of site D and 41 of site $\mathrm{E}$ ) with the most frequent stage being found were microfilariae (mfs) (84.4\%) (See additional file 1: Microfilaria of Cercopithifilaria sp. in ticks), followed by developing L1 (11.1\%), L2 (6.7\%) and L3 (6.7\%) (Table 4). As many as 1469 larvae (i.e., $1453 \mathrm{mfs}, 11 \mathrm{~L} 1$ and 5 L2) of Cercopithifilaria sp. were found in a single tick (See additional file 2: Second stage larvae of Cercopithifilaria sp. in ticks). Interestingly, 16 (11 males and 5 females) out of the 38 ticks harbouring the $\mathrm{mfs}$ stage of Cercopithifilaria sp. were collected from dogs negative by microscopical examination of skin sediment.

\section{Discussion}

This study represents the first survey to investigate the occurrence of this recently discovered Cercopithifilaria

Table 2 Agreement between diagnostic tests: number of positive and negative samples examined in parallel by microscopical examination of skin sediment and molecular analysis of skin for Cercopithifilaria sp. infection in Sicily (Site E) and Spain (Site F)

\begin{tabular}{lllll}
\hline & Microscopical examination & \multicolumn{2}{l}{$\begin{array}{l}\text { Molecular } \\
\text { examination }\end{array}$} & \multirow{2}{*}{ Total } \\
\cline { 3 - 4 } & & Positive & Negative & \\
\hline Sicily (E) & Positive & 6 & 9 & 15 \\
& Negative & 16 & 82 & 98 \\
& Total & 22 & 91 & 113 \\
\hline \multirow{5}{*}{ Microscopical examination } & Molecular & Total \\
\cline { 3 - 4 } & & examination & \\
\cline { 2 - 4 } & & Positive & Negative & \\
\hline Spain (F) & Positive & 2 & 1 & 3 \\
& Negative & 3 & 5 & 8 \\
& Total & 5 & 6 & 11 \\
\hline
\end{tabular}

sp. in dogs from selected geographical areas of the Mediterranean basin and in ticks collected from the same animals. Indeed, as a main conclusion for the first report of the Cercopithifilaria sp. with dermal microfilariae in a dog from Sicily [12], it was not clear whether this was an occasional finding or a widespread, though neglected, canine infestation. Nonetheless, considering the widespread distribution of $R$. sanguineus [28], which is regarded as the putative vector of this filarioid [15], it was suggested that this species could be a common parasite of dogs in temperate areas. Accordingly, this study indicates that the Cercopithifilaria sp. here investigated is widespread in dogs from the Mediterranean countries surveyed with an overall prevalence ranging from $10.5 \%$ to $13.9 \%$. These prevalence rates are higher than those detected in large epidemiological surveys for other filarioids with the exception of $D$. repens in dogs living in hyper-endemic areas of southern Italy, where a prevalence of microfilaraemic dogs of up to $30.8 \%$ were recorded [29]. In particular, the highest prevalence rate of infested animals was recorded in Spain (from 21.6\% up to $45.5 \%$ by microscopical examination or molecular detection of skin samples) whereas the prevalence of infestation in Italy (up to 19.5\%) was close to the mean values. The significant difference in the frequency of infection in male dogs $(23.4 \%)$ compared to females (7.5\%) found in site A might reflect the male-biased infestation by $R$. sanguineus in some areas, as demonstrated in a confined dog population in southern Italy [21].

The cumulative incidences of $7.7 \%$ over ten months and $13.9 \%$ over seven months were recorded in sites $\mathrm{B}$ and $\mathrm{C}$, respectively. In both sites incidence was calculated after the spring and summer seasons, which are the seasons with the highest number of $R$. sanguineus ticks infesting animals in the Mediterranean region and, specifically, in the study sites [21]. Interestingly, even if dogs from site $C$ were exposed to ticks for a shorter period than dogs from site $\mathrm{B}$, a high incidence rate was detected in site $C$. This finding could be explained by the higher rate of Cercopithifilaria sp. infestation in dogs from site C (12.5\%) than that from site B $(5.3 \%)$. However, both cumulative incidence values recorded in this study were very high if compared to the force of infection, which expresses the probability of a susceptible dog becoming infected in one year, calculated across European countries for D. immitis (up to 8.4\%) and D. repens (up to $3.3 \%$ ), respectively [30].

A low level of agreement between the two diagnostic methods (i.e., microscopical examination versus PCR) was recorded in this study, considering either skin samples or ticks. In addition, in all ticks and skin samples from sites D and $G$ that were tested in parallel, the rate of infestation was higher when determined by 
Table 3 Association between Cercopithifilaria sp. infestation in dogs (positive/negative) and the presence or absence of tick infestation on the same animals from the different sites examined

\begin{tabular}{|c|c|c|c|c|c|c|c|}
\hline \multirow[t]{2}{*}{ Locality/region } & \multirow[t]{2}{*}{$N$} & \multicolumn{2}{|c|}{ Tick-infested dogs } & \multicolumn{2}{|c|}{ Tick-free dogs } & \multirow[t]{2}{*}{ Odds ratio $(95 \% \mathrm{Cl})$} & \multirow[t]{2}{*}{$P$} \\
\hline & & positive & negative & positive & negative & & \\
\hline Putignano (C) & 80 & 6 & 24 & 4 & 46 & $2.9(0.7-11.2)$ & 0.1640 \\
\hline Basilicata (D) & 50 & 5 & 13 & 1 & 31 & $11.9(1.3-112.3)$ & 0.0184 \\
\hline Sicilia (E) & 113 & 13 & 28 & 2 & 70 & $16.2(3.4-76.7)$ & $<0.0001$ \\
\hline Madrigal de la Vera (F) & 51 & 9 & 32 & 2 & 8 & $1.1(0.2-6.3)$ & 1.000 \\
\hline Xanthi (G) & 23 & 1 & 8 & 0 & 14 & $5.1(0.2-14.4)$ & 0.3913 \\
\hline
\end{tabular}

microscopical examination than by PCR. This discordance might be due to the fact that all microfilariae present in the skin samples had deposited on the bottom of the eppendorf tubes, being easily detected by microscopical examination of the sediment. Consequently, the skin piece used for DNA extraction and subsequent PCR testing had less or no microfilariae. Similarly, all developing larvae detected in ticks were isolated, singularly observed, photographed and thus the remaining tissues of the dissected tick that was used for molecular testing had less or no microfilariae as well.

The occurrence of developing second and third-stage larvae of Cercopithifilaria sp. in R. sanguineus after feeding on an infested dog, their morphological similarity with those of Cercopithifilaria genus $[13,16,17]$ and molecular homology with the dermal microfilariae of

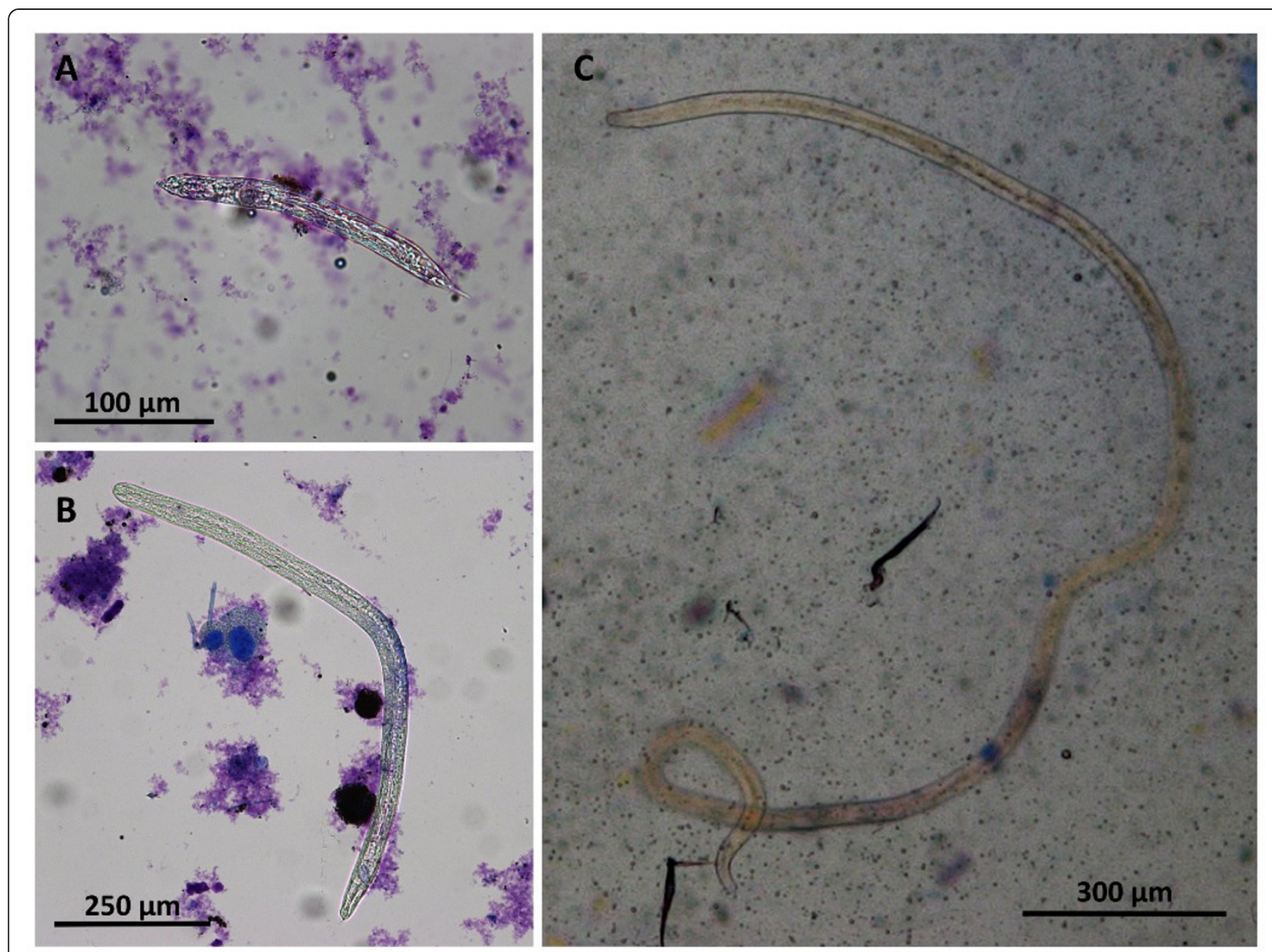

Figure 2 Larval Cercopithifilaria sp. in dissected ticks. Developing forms of Cercopithifilaria sp. found in dissected ticks: A) First stage larva; B) Second stage larva; C) Third stage infective larva. Ticks were dissected in few drops of saline solutions and larvae stained with methylene blue (1\%). 
Table 4 Number and percentage of infected ticks, mean intensity, range of infestation and measurements of larval Cercopithifilaria sp. according to their developmental stage

\begin{tabular}{|c|c|c|c|c|c|}
\hline \multirow[t]{2}{*}{ Developing forms } & \multirow[t]{2}{*}{$\%$ Tick infected (pos/tot) } & \multirow[t]{2}{*}{ Mean intensity ( \pm S.D.) } & \multirow[t]{2}{*}{ Range } & \multicolumn{2}{|c|}{ Measurements (microns) } \\
\hline & & & & Length ( \pm S.D.) & Width ( \pm S.D.) \\
\hline mfs & $84.4(38 / 45)$ & $49.6( \pm 237.5)$ & $1-1453$ & $192.2( \pm 9.9)$ & $5.6( \pm 1)$ \\
\hline L1 & $11.1(5 / 45)$ & $6.8( \pm 6.2)$ & $1-15$ & $229.2( \pm 16.6)$ & $19.2( \pm 4.4)$ \\
\hline L2 & $6.7(3 / 45)$ & $12.3( \pm 13.7)$ & $2-27$ & $724( \pm 13.6)$ & $27.1( \pm 1)$ \\
\hline L3 & $6.7(3 / 45)$ & $8.3( \pm 12.7)$ & $1-23$ & $1706( \pm 61)$ & $26.5( \pm 1.5)$ \\
\hline
\end{tabular}

Tick samples constituted a total of 45 positive ticks collected in Basilicata (Site D) and Sicily (Site E). Measurements of length and width of developing larvae in ticks are expressed as mean calculated on 10 microfilariae (mfs), 7 L1, 7 L2 and 5 L3.

Cercopithifilaria sp. described from the same dog [12] suggested that this tick species may represent a competent intermediate host [15]. The positivity for Cercopithifilaria sp. recorded in ticks collected from sampled dogs (from 5.2 to $16.7 \%$ and from 0.5 to $3.9 \%$ by using dissection and PCR), provides circumstantial evidence indicating that $R$. sanguineus is the vector for this filarioid under natural conditions. Analogously, in sites where the highest number of ticks was collected on dogs (i.e., site D and E) tick infestation was significantly associated with Cercopithifilaria sp. infestation (Table 3 ). These data along with the evidence of the presence of $\mathrm{mfs}$ and developing second- and third-stage larvae in ticks, further indicate that $R$. sanguineus may act as vector of this Cercopithifilaria sp. in nature.

Indeed, all developing forms found in ticks were similar to the different stages described elsewhere for this Cercopithifilaria sp. [15]. Interestingly, the extraordinary finding of up to 1469 developing forms of Cercopithifilaria sp. in one tick (i.e., $1453 \mathrm{mfs}, 11 \mathrm{~L} 1$ and $5 \mathrm{~L} 2$ ) suggested that Cercopithifilaria sp. infestation is well tolerated by $R$. sanguineus and that the tick viability is not impaired by the nematode larvae. This could represent the foundation for further investigations on the Cercopithifilaria sp.-vector relationship and to explain the broad distribution of this nematode among tickinfested dogs. The finding that both male and female ticks positive for Cercopithifilaria sp. were collected from both positive $(38 / 90,42.2 \%)$ and negative (52/90, $57.8 \%)$ dogs might indicate that both sexes act as vectors of this nematode. In particular, the multiple-feeding behaviour of male ticks on the same dog or on cohoused dogs and the long periods of time they spend on the host [31] increase their potential as vectors of pathogens (e.g., Ehrlichia canis, ref. 32), including Cercopithifilaria sp.

Our data also suggest that the xenodiagnosis (i.e., detection of larvae in their vectors) might be useful for detecting skin-dwelling microfilariae and should even be preferred to the direct observation of skin samples, if the aim of study is to detect the occurrence of this parasite in a given animal population. Whereas, the examination of skin samples should be preferred when the diagnosis is requested at the individual level. In addition, in post mortem studies, soaking carcasses and skin strips in warm saline (soon after the animal death) followed by a series of decantation, is efficacious in retrieving adult and larval worms [33]. However, this procedure is not easy to perform, it is time consuming, and not acceptable for the majority of owners.

\section{Conclusions}

Finally, the scientific evidence presented here suggests that, in addition to the most common species of filarioids known to infest dogs (i.e., D. immitis, D. repens and $A$. reconditum), Cercopithifilaria sp. with dermal microfilariae should also be considered. Although microfilariae are useful to morphologically differentiate onchocercid species, no definitive conclusion on the identity of this species can be made in the absence of other nematode stages, particularly adults. Nonetheless, the microfilariae here retrieved are morphologically close to those of C. bainae (i.e., $185.18 \mu \mathrm{m}$ and $6.59 \mu \mathrm{m}$ in length and width, respectively [34]). Future studies should prioritize investigations on the specific identity of this parasite, its pathogenicity at a local (dermal) and/ or systemic level and the potential for antigenic crossreactivity with other onchocercids of dogs. Undoubtedly, the genetic make-up of Cercopithifilaria spp. and their genetic affiliation with the vector and the host might provide interesting information on the role $R$. sanguineus in disseminating Cercopithifilaria onchocercids within animal populations.

\section{Additional material}

Additional file 1: Microfilaria of Cercopithifilaria sp. in ticks Microfilaria of Cercopithifilaria sp. found in a dissected tick.

Additional file 2: Second stage larvae of Cercopithifilaria sp. in ticks Second stage larvae of Cercopithifilaria sp. found at tick dissection. Note the presence in the same field of numerous microfilariae of the same species.

\section{Acknowledgements}

The authors thank Alessandro Fogliazza (Merial, Italy), Frederic Buegnet and Lénaïg Halos (Merial, France) for partially supporting this research and 
Dorothe Stanneck and Norbert Mencke (Bayer Animal Health $\mathrm{GmbH}$ ) for sponsoring previous research from where many tested samples derived.

\section{Author details}

'Dipartimento di Sanità Pubblica e Zootecnia, Università degli Studi di Bari, Valenzano, BA, Italy. ${ }^{2}$ Dipartimento di Sanità Pubblica Veterinaria, Facoltà di Medicina Veterinaria, Università degli Studi di Messina (Messina), Italy. ${ }^{3}$ Department of Infectious and Parasitic Diseases and Pathology, Faculty of School of Veterinary Medicine, Aristotle, University of Thessaloniki, Thessaloniki 54 124, Greece. ${ }^{4}$ Departamento de Sanidad Animal, Facultad de Veterinaria, Universidad Complutense de Madrid, Spain. ${ }^{5}$ Département Systématique et Evolution, UMR 7205 CNRS, Muséum National d'Histoire Naturelle, Paris, France.

\section{Authors' contributions}

DO, conceived the research, collected samples, contributed with data analysis and interpretation and wrote the first draft of the manuscript. EB, FDT, collected samples, contributed with data analysis, interpretation and revision of the manuscript. $O B$, contributed with data analysis, interpretation and revision of the manuscript. MSL, SW and GA run the molecular assays. GM and EP collected samples, contributed with data analysis and interpretation and revision of the manuscript. RPL, GG and EN collected samples and dissected ticks. All authors read and approved the final version of the manuscript.

\section{Competing interests}

The authors declare that they have no competing interests.

Received: 3 October 2011 Accepted: 3 January 2012

Published: 3 January 2012

\section{References}

1. Sréter T, Széll Z: Onchocercosis: a newly recognized disease in dogs. Vet Parasitol 2008, 151:1-13.

2. Orihel TC, Ash LR, Holshuh HJ, Santenelli S: Onchocerciasis in a California dog. Am J Trop Med Hyg 1991, 44:513-517.

3. Eberhard ML, Ortega Y, Dial S, Schiller CA, Sears AW, Greiner E: Ocular Onchocerca infections in two dogs in western United States. Vet Parasitol 2000, 90:333-338.

4. Zarfoss MK, Dubielzig RR, Eberhard ML, Schmidt KS: Canine ocular onchocerciasis in the United States: two new cases and a review of the literature. Vet Ophthalmol 2005, 8:51-57.

5. Széll Z, Erdélyi I, Sréter T, Albert M, Varga I: Canine ocular onchocercosis in Hungary. Vet Parasitol 2001, 97:243-249.

6. Komnenou A, Eberhard ML, Kaldrymidou E, Tsalie E, Dessiris A: Subconjunctival filariasis due to Onchocerca sp. in dogs: report of 23 cases in Greece. Vet Ophthalmol 2002, 5:119-126.

7. Hermosilla C, Hetzel U, Bausch M, Grübl J, Bauer C: First autochthonous case of canine ocular onchocercosis in Germany. Vet Rec 2005, 156:450-452.

8. Sréter-Lancz Z, Széll Z, Sréter T: Molecular genetic comparison of Onchocerca sp. infecting dogs in Europe with other spirurid nematodes including Onchocerca lienalis. Vet Parasitol 2007, 148:365-370.

9. Otranto D, Eberhard ML: Zoonotic helminths affecting the human eye. Parasit Vectors 2011, 4:41.

10. Otranto D, Sakru N, Testini G, Gürlü VP, Yakar K, Lia RP, Dantas-Torres F, Bain O: Case report: First evidence of human zoonotic infection by Onchocerca lupi (Spirurida, Onchocercidae). Am J Trop Med Hyg 2011, 84:55-58.

11. Bain O, Uni S, Takaoka H: A synthetic look at a twenty years old taxon, Cercopithifilaria its probable evolution. In Proceedings of the 10th International Congress of Parasitology (ICOPA): 4-9 August 2002; Vancouver (Canada) Edited by: Monduzzi Editore 2002, 365-368.

12. Otranto D, Brianti E, Dantas-Torres F, Weigl S, Latrofa MS, Gaglio G, Cauquil L, Giannetto S, Bain O: Morphological and molecular data on a Cercopithifilaria species from a dog in Sicily. Vet Parasitol 2011, 182:221-229.

13. Noè G: Contribuzioni alla Sistematica e alla Anatomia del Genere Filaria. La Filaria grassii Roma, Istituto di Anatomia Comparata della Regia Università di Roma; 1907, 236-252.
14. Almeida GLG, Vicente JJ: Cercopithifilaria bainae sp. n. parasita de Canis familiaris (L.) (Nematoda, Filarioidea). Atas Soc Biol Rio de Janeiro 1984, 24:18.

15. Brianti E, Otranto D, Dantas-Torres F, Weigl S, Latrofa MS, Gaglio G, Napoli E, Brucato G, Cauquil L, Giannetto S, Bain O: Rhipicephalus sanguineus (Ixodida, Ixodidae) as intermediate host of a canine neglected filarial species with dermal microfilariae. Vet Parasitol 2012, 10:330-7.

16. Bain O, Aeschlimann A, Chatelanat P: Présence, chez des tiques de la région de Genève, de larves infestantes qui pourraient se rapporter à la filaire de chien Dipetalonema grassii. Ann Parasitol Hum Comp 1982, 57:643-646.

17. Pampiglione S, Canestri Trotti G, Marchetti S: Ritrovamento di Diptalonema grassii (Noè, 1907) in Rhipicephalus sanguineus su cane in Italia e descrizione di alcuni suoi stadi larvali. Parassitologia 1983, 25:316.

18. Otranto D, Paradies P, Lia RP, Latrofa MS, Testini G, Cantacessi C, Mencke N, Galli G, Capelli G, Stanneck D: Efficacy of a combination of $10 \%$ imidacloprid $/ 50 \%$ permethrin for the prevention of leishmaniasis in kennelled dogs in an endemic area. Vet Parasitol 2007, 144:270-278.

19. Otranto D, de Caprariis D, Lia RP, Tarallo V, Lorusso V, Testini G, DantasTorres F, Latrofa S, Diniz PP, Mencke N, Maggi RG, Breitschwerdt E, Capelli G, Stanneck D: Prevention of endemic canine vector-borne diseases using imidacloprid $10 \%$ and permethrin $50 \%$ in young dogs: a longitudinal field study. Vet Parasitol 2010, 172:323-332.

20. Brianti E, Pennisi MG, Brucato G, Risitano AL, Gaglio G, Lombardo G, Malara D, Fogliazza A, Giannetto S: Efficacy of the fipronil 10\%+(S)methoprene $9 \%$ combination against Rhipicephalus sanguineus in naturally infested dogs: speed of kill, persistent efficacy on immature and adult stages and effect of water. Vet Parasitol 2010, 17:96-103.

21. Lorusso V, Dantas-Torres F, Lia RP, Tarallo VD, Mencke N, Capelli G, Otranto D: Seasonal dynamics of the brown dog tick, Rhipicephalus sanguineus, on a confined dog population in Italy. Med Vet Entomol 2010, 24:309-315.

22. Dantas-Torres F, Figueredo LA, Otranto D: Seasonal variation in the effect of climate on the biology of Rhipicephalus sanguineus in southern Europe. Parasitology 2011, 138(Suppl 4):527-536.

23. Dantas-Torres F: The brown dog tick, Rhipicephalus sanguineus (Latreille, 1806) (Acari: Ixodidae): from taxonomy to control. Vet Parasitol 2008, 152:173-185.

24. Sangioni LA, Horta MC, Vianna MC, Gennari SM, Soares RM, Galvão MA, Schumaker T, Ferreira F, Vidotto O, Labruna MB: Rickettsial infection in animals and Brazilian spotted fever endemicity. Emerg Infect Dis 2005, 11:265-270.

25. Otranto D, Testini G, Dantas-Torres F, Latrofa MS, Diniz PP, de Caprariis D, Lia RP, Mencke N, Stanneck D, Capelli G, Breitschwerdt EB: Diagnosis of canine vector-borne diseases in young dogs: a longitudinal study. J Clin Microbiol 2010, 48:3316-3324.

26. Larkin MA, Blackshields G, Brown NP, Chenna R, McGettigan PA, McWilliam H, Valentin F, Wallace IM, Wilm A, Lopez R, Thompson JD, Gibson TJ, Higgins DG: ClustalW and ClustalX version 2. Bioinformatics 2007, 23:2947-2948.

27. Thrusfield M: Veterinary Epidemiology. 2 edition. Blackwell Scientific Publication, Oxford; 1995.

28. Dantas-Torres F: Biology and ecology of the brown dog tick, Rhipicephalus sanguineus. Parasit Vectors 2010, 3:26.

29. Giannetto S, Poglayen G, Gaglio G, Brianti E: Prevalence and epidemiological aspects of microfilaraemia in dogs in Sicily. Abstract book of the 1st European Dirofilaria days 2007, 5, 22-25 February 2007; Zagreb, Croatia. Edit by Albert Marinculic.

30. Trotz-Williams LA, Trees AJ: Systematic review of the distribution of the major vector-borne parasitic infections in dogs and cats in Europe. Vet Rec 2003, 157:97-105.

31. Little SE, Hostetler J, Kocan KM: Movement of Rhipicephalus sanguineus adults between co-housed dogs during active feeding. Vet Parasitol 2007, 150:139-145.

32. Bremer WG, Schaefer JJ, Wagner ER, Ewing SA, Rikihisa Y, Needham GR, Jittapalapong S, Moore DL, Stich RW: Transstadial and intrastadial experimental transmission of Ehrlichia canis by male Rhipicephalus sanguineus. Vet Parasitol 2005, 131(1-2):95-105.

33. Eberhard ML: Dipetalonema (Cercopithifilaria) kenyensis subgen. et sp. $\mathrm{n}$. (Nematoda: Filarioidea) from African baboons, Papio anubis. J Parasitol $1980,66: 551-554$. 
34. Almeida GLG, Vicente JJ: Dipetalonema reconditum (Grassi, 1890), Dipetalonema grassii (Noè, 1907) e Dirofilaria immitis (Leidy, 1856) em cães na cidade do Rio de Janeiro (Nematoda - Filarioidea). Atas Soc Biol Rio de Janeiro 1982, 23:9-12.

doi:10.1186/1756-3305-5-1

Cite this article as: Otranto et al:: On a Cercopithifilaria sp. transmitted by Rhipicephalus sanguineus: a neglected, but widespread filarioid of dogs. Parasites \& Vectors 2012 5:1.

Submit your next manuscript to BioMed Central and take full advantage of:

- Convenient online submission

- Thorough peer review

- No space constraints or color figure charges

- Immediate publication on acceptance

- Inclusion in PubMed, CAS, Scopus and Google Scholar

- Research which is freely available for redistribution

Submit your manuscript at www.biomedcentral.com/submit 